Full length article

\title{
Molecular diversity and evolution of defensins in the manila clam Ruditapes philippinarum
}

\author{
Qing Wang a , Linbao Zhang ${ }^{\mathrm{b}}$, Dinglong Yang ${ }^{\mathrm{a}, \mathrm{c}}$, Qian Yu ${ }^{\mathrm{a}, \mathrm{c}}$, Fei Li ${ }^{\mathrm{a}}$, Ming Cong ${ }^{\mathrm{a}}$, \\ Chenglong $\mathrm{Ji}^{\mathrm{a}}$, Huifeng $\mathrm{Wu}^{\mathrm{a}}$, Jianmin Zhao ${ }^{\mathrm{a},{ }^{\mathrm{c}}}$, \\ ${ }^{a}$ Key Laboratory of Coastal Environmental Processes and Ecological Remediation, CAS, Shandong Provincial Key Laboratory of Coastal Zone Environmental \\ Processes, Yantai Institute of Coastal Zone Research, Chinese Academy of Sciences, Yantai 264003, PR China \\ b South China Sea Fisheries Research Institute, Chinese Academy of Fishery Sciences, Guangzhou 510300, PR China \\ ${ }^{\mathrm{c}}$ University of Chinese Academy of Sciences, Beijing 100049, PR China
}

\section{A R T I C L E I N F O}

\section{Article history:}

Received 22 April 2015

Received in revised form

31 August 2015

Accepted 2 September 2015

Available online 9 September 2015

\section{Keywords:}

Defensin

Manila clam

Diversity

Positive selection

\begin{abstract}
A B S T R A C T
Four types of defensins were identified in Manila clam and designated as Rpdef1, Rpdef2, Rpdef3 and Rpdef4, which encoded a polypeptide of $49,46,45$ and 42 amino acids, respectively. Sequence alignments indicated that Rpdef1 shared $46.9 \%$ identity with Rpdef2, $40.8 \%$ with Rpdef3, and $34.7 \%$ with Rpdef4. Analysis of transcript polymorphism showed that Rpdef3 accounted for about $60 \%$ frequency of Rpdefs occurrence in clams from three geographic origins (Dalian, Qingdao and Hangzhou). By quantitative real-time RT-PCR (qRT-PCR) analysis, the transcripts of Rpdefs were mainly detected in hemocytes and they responded sensitively to bacterial challenge in hemocytes. Evolutionary analysis indicated that all Rpdefs were under positive selection with positively selected basic amino acid residues detected in the C-terminal regions, which perhaps have a functional relevance by modifying the charge distribution of Rpdefs. The results also showed some lineages with $\mathrm{dN} / \mathrm{dS}>1$, suggesting positive selection pressures existed in some lineages of phylogeny tree constructed by mollusk defensins. Overall, our results suggest that Rpdefs perhaps played important roles in host defense and positive selection is the major driving force in generating high diversity of defensins in the Manila clam.
\end{abstract}

() 2015 Elsevier Ltd. All rights reserved.

\section{Introduction}

Invertebrates exclusively depend on their innate immunity which consists of both cellular and humoral defenses [1,2]. The former includes phagocytosis or encapsulation of pathogens with subsequent pathogen destruction via enzyme activity and oxygen metabolite release, while the latter includes various reactions mediated by molecules such as antimicrobial peptides (AMPs) and proteins $[3,4]$. In marine invertebrates, AMPs represent the major humoral defense system against infection. The modes of action by which AMPs kill bacteria are diverse, and most of them related to plasma membrane disturbance and lethal alteration of microbial integrity [5]. In marine mollusks, several kinds of AMPs have been characterized and studied, including defensins, mytilins, myticins, mytimycin, big defensins and mytimacins [6-13].

Among the large number of AMPs, defensin is one of the most

\footnotetext{
* Corresponding author

E-mail address: jmzhao@yic.ac.cn (J. Zhao).
}

ubiquitous families [1]. Defensins are a collection of small cationic peptides with molecular weights of approximately 3-5 kDa [14]. Generally, the animal defensin molecules can be classified into four major groups according to their structure and origin: $\alpha$-defensin, $\beta$ defensin, $\theta$-defensin and invertebrate defensin [15]. These defensins display similar structural features: the presence of a signal peptide at the $\mathrm{N}$-terminal region, followed by the mature peptide region which is characterized by $6-8$ conserved cysteine residues forming three or four disulfide bonds, and a C-terminal extension rich in anionic residues [16]. Defensins have been found to be widely distributed in marine invertebrate animals, especially in mollusks. Presently, multiple defensin molecules have been successively identified from mussels, oysters, clam and abalone [14,17-20]. It has been shown that defensins from marine mollusks are active against Gram-positive and Gram-negative bacteria and fungi, suggesting that they play important roles in innate immune response of mollusks $[6,7,17,18,21,22]$.

Due to their direct interaction with altered/new pathogens, AMPs exhibit an extraordinary diversity in their structure and 
function $[23,24]$. Molecular diversity of AMPs such as myticin and defensin has been detected in marine mussels and oysters $[8,25,26]$. Recently, the defensin from freshwater pearl mussel Hyriopsis cumingii has also been shown to contain six isoforms [27]. Sequence diversification of AMPs by gene duplication has been reported for both vertebrates and invertebrates [28,29]. Moreover, an increasing number of studies suggested that the evolution of AMPs is driven by positive selection in both vertebrates and invertebrates [26,30-32].

Although the knowledge on mollusk defensins has been much reported, the information on evolutionary pattern of mollusk defensins is still very limited. In this study, four isoforms of defensins have been characterized from the Manila clam and their biochemical properties and structures have been predicted. Moreover, the evolutionary patterns of these defensins from Manila clam and other mollusks have also been discussed.

\section{Materials and methods}

\subsection{Animal culture and challenge}

For mRNA polymorphism characterization, the clams Ruditapes philippinarum (shell length: $\sim 3.0-4.0 \mathrm{~cm}$ ) were purchased from culture farms at three different sites (Dalian, Qingdao and Hangzhou) (Fig. 1). The clams are acclimated for two week before commencement of the experiment. They were maintained in filtered seawater at $20-22{ }^{\circ} \mathrm{C}$ and $30 \%$ salinity throughout the whole experiment. Then sixty clams of three geographic origins (20 individuals for each location) were immersed with high density of live Micrococcus luteus and Vibrio anguillarum with a final concentration of $1 \times 10^{7} \mathrm{CFU} \mathrm{mL}{ }^{-1}$ respectively. After $24 \mathrm{~h}$ of challenge, the hemocytes, digestive glands and gills of 45 individuals (15 individuals for each location) were sampled and stored in liquid nitrogen before use.

For bacterial challenge experiment, adult clams (shell-length: $\sim 3.5-4.5 \mathrm{~cm}$ ) were purchased from a local culturing farm (Yantai) and acclimatized for 7 days. Then the clams were exposed to $V$. anguillarum at a final concentration of $1 \times 10^{7} \mathrm{CFU} \mathrm{mL}^{-1}$. At $12 \mathrm{~h}$, $24 \mathrm{~h}$ and $48 \mathrm{~h}$ intervals following the challenge, the hemocytes of four individuals were sampled and stored in liquid nitrogen. Meanwhile, the hemocytes, gill, digestive gland, mantle and foot of four untreated clams were also sampled to determine the tissue-distribution profiles of Rpdefs.

\subsection{Total RNA extraction and sequence amplification}

Frozen tissues were pulverized under liquid nitrogen, and subjected to total RNA extraction using the TRIzol Reagent (Invitrogen, USA). The extracted RNA was then treated with RQ1 RNase-Free DNase (Promega, USA) to remove DNA contamination. Singlestranded cDNA was synthesized from the total RNA with M-MLV reverse transcriptase (Promega, USA).

The EST sequences from cDNA library constructed from Manila clam hemocytes (unpublished) were used to construct a blast

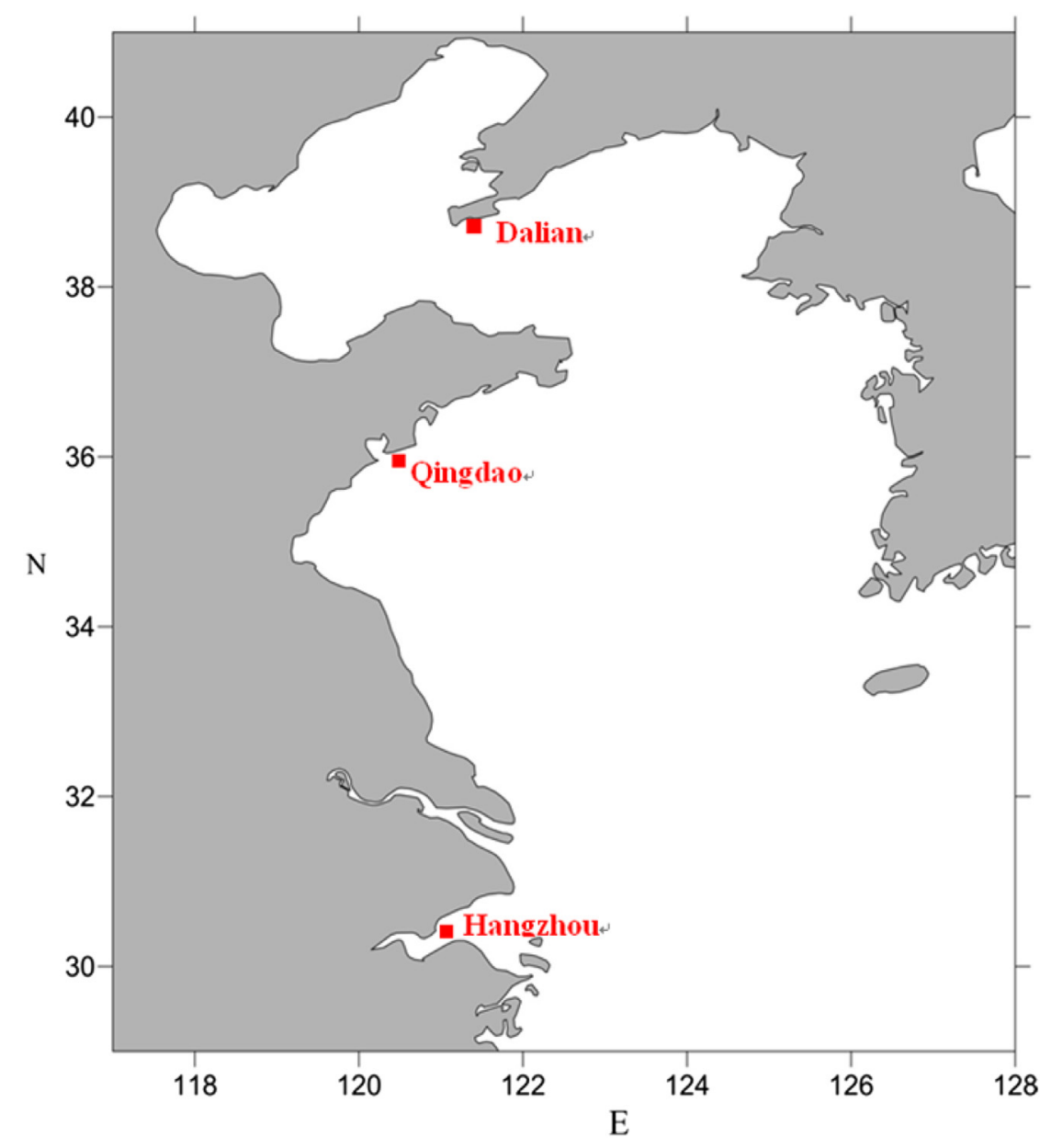

Fig. 1. The sampling sites of manila clam Ruditapes philippinarum along the coast of China. 
Table 1

Primers used in the present study.

\begin{tabular}{lll}
\hline Primer & Sequence $\left(5^{\prime}-3^{\prime}\right)$ & Sequence information \\
\hline P1 (reverse) & TGGTGCTGTGATGAGTTCTAT' & $5^{\prime}$ RACE primer \\
P2 (reverse) & TGCACCTCTGACGTAATGT & $5^{\prime}$ RACE primer \\
P3 (forward) & GGTTTGGTTGCCCTGAAGATGA & $3^{\prime}$ RACE primer \\
P4 (forward) & GAACTCATCACAGCACCAACA & $3^{\prime}$ RACE primer \\
P5 (forward) & CAACAGGTTTAGCACTCAACGG & Polymorphism detection primer \\
P6 (reverse) & AAACTTGCTTGCGTGTTGGTGC & Polymorphism detection primer \\
P7 (forward) & TTGATGCCGGGTTTGGTTG & Real time primer for Rpdef1 \\
P8 (reverse) & CAACCGTAACAAGTGCACCT & Real time primer for Rpdef1 \\
P9 (forward) & CCGAAAATGGCTGCCCTAAT & Real time primer for Rpdef2 \\
P10 (reverse) & GTTACACAGGCACGACAAGT & Real time primer for Rpdef2 \\
P11 (forward) & AGGACGATGATTGCTTTTACTGT & Real time primer for Rpdef3 \\
P12 (reverse) & CCCAGCAATCGTTACACCTG & Real time primer for Rpdef3 \\
P13 (forward) & CGTTGATGGCTGTCGTGTAT & Real time primer for Rpdef4 \\
P14 (reverse) & CGAGCAAGCGTAACACCTG & Real time primer for Rpdef4 \\
P15 (forward) & CTCCCTTGAGAAGAGCTACGA & Real time primer for $\beta$-actin \\
P16 (reverse) & GATACCAGCAGATTCCATACCC & Real time primer for $\beta$-actin \\
\hline
\end{tabular}

database using the makeblastdb program available from the NCBI website. The oyster defensin sequence (GenBank accession no. CAJ19280) was used as a query sequence in a tblastn search (default parameters, version 2.2.28+) against this database to identify homologues within the database. Then a putative Manila defensin sequence was identified and subjected to blastx searches against the NCBI nucleotide database, to confirm its identity. To generate the full-length cDNA of $R$. philippinarum genes (Rpdefs), two reverse primers P1 and P2, and two forward primers P3 and P4 (Table 1), were designed based on the EST sequence. The nested PCR strategy was applied to the $3^{\prime}$ and $5^{\prime}$ RACE. For transcript polymorphism detection, two specific primers (P5 and P6, Table 1) designed in the $5^{\prime}$ UTR and 3'UTR were employed to clone the full coding sequence of Rpdefs. The PCR profile and subsequent sequencing were conducted as described previously $[33,34]$. A total of 126 positive Rpdef clones were bi-directionally sequenced respectively.

\subsection{Quantitative real-time PCR ( $q R T-P C R)$ assay}

Quantitative real-time PCR (qRT-PCR) was carried out in an ABI 7500 Real-time Detection System by using the SYBR ExScript qPCR Kit (Takara, Japan) as described previously [33]. The PCR amplification was carried out in a total volume of $50 \mu \mathrm{L}$, containing $25 \mu \mathrm{L}$ of $2 \times$ SYBR Green PCR Master Mix, $20 \mu \mathrm{L}$ of the diluted cDNA, $1 \mu \mathrm{L}$ of each of primers ( $10 \mu \mathrm{mol} / \mathrm{L}$ ), and $3 \mu \mathrm{L}$ of DEPC-treated water. The thermal profile for qPCR was $50{ }^{\circ} \mathrm{C}$ for $2 \mathrm{~min}, 95{ }^{\circ} \mathrm{C}$ for $10 \mathrm{~min}$ followed by 40 cycles of $95{ }^{\circ} \mathrm{C}$ for $15 \mathrm{~s}$ and $60^{\circ} \mathrm{C}$ for $1 \mathrm{~min}$. All reactions were run in triplicate. Dissociation curve analysis of amplicons was performed at the end of each PCR reaction to confirm that only one PCR product was amplified and detected. The expressions of Rpdefs were analyzed using the $2^{-\Delta \Delta \mathrm{CT}}$ method with $\beta$-actin gene as the internal control. The primers used to quantify the expression of Rpdefs were listed in Table 1.

\subsection{Sequence analysis, structure prediction and phylogenetic analysis}

The searches for nucleotide and protein sequence similarities were performed with the BLAST algorithm (http://www.ncbi.nlm. nih.gov/blast). The deduced protein sequences were analyzed with ExPASy (http://www.expasy.org/). Signal peptide was predicted by SignalP 4.0 server (http://www.cbs.dtu.dk/services/ SignalP/). Prediction of putative disulfide bonds was performed using Scratch Protein Predictor (http://scratch.proteomics.ics.uci. edu/), DISULFIND web-server (http://cassandra.dsi.unifi.it/) and DiANNA web server (http://clavius.bc.edu/ clotelab/DiANNA/). The
3D structure of defensins was predicted with Phyre 2 server (Protein Homology/analogY Recognition Engine V 2.0), and visualized using the PyMOL software (DeLano, The PyMOL Molecular Graphics System, 2002, http://www.pymol.org). Multiple alignments were performed with the ClustalW program (http://www.ebi.ac.uk/ Tools/msa/clustalw2/). A maximum likelihood (ML) phylogenetic tree based on the nucleotide sequences of mollusk defensins was constructed using PhyML 3.0 [35]. For phylogenetic analyses, the optimum evolutionary models were selected using the jModelTest program [36]. For ML analysis, 100 bootstraps were used to estimate the node reliability.

\subsection{Testing for positive selection}

The nucleotide sequences encoding amino acids of Rpdefs were used to construct a Maximum likelihood (ML) tree using appropriate nucleotide substitution model. The reliability of interior branches of each phylogeny was assessed with 1000 bootstraps. The phylogeny was used to estimate nonsynonymous to synonymous substitution rate ratio $(\omega=\mathrm{dN} / \mathrm{dS})$ by the maximum likelihood (ML) method implemented in CODEML program of the PAML 4.4 software package [37]. Positive selection can be inferred from a higher proportion of nonsynonymous than synonymous substitutions per site $(\mathrm{dN} / \mathrm{dS}>1)$. Likelihood ratio tests (LRTs) were used to determine whether any codon positions were subjected to positive selection as indicated by $\omega>1$.

To test for heterogeneous selective pressure at amino acid sites, the site-specific models were tested: M0 (one-ratio) against M3 (discrete), M1a (nearly neutral) against M2a (positive selection), M7 (beta) against M8 (beta \& $\omega$ ). The assumption and parameters of each model were as describe previously [33]. The branch model was also conducted using the likelihood ratio test between the oneratio model and the free-ratio model results to detect positive selection acting on particular lineages of the phylogenetic tree. The $\omega$ value in the one-ratio model was fixed whereas the value in the free-ratio model was estimated. The LRTs between nested models were conducted by comparing twice the difference of the loglikelihood values $(2 \Delta \mathrm{L})$ between two models with the $\chi^{2}$ distribution. The Naive Empirical Bayes (NEB) method and Bayes empirical Bayes (BEB) method were used to calculate the posterior probability that each codon was from the site class of positive selection under models M3, M2a and M8 respectively [38].

\subsection{Statistical analysis}

SPSS 16.0 software (SPSS Inc., USA) was used for statistical 
analysis. All data were given in terms of relative mRNA expression as means $\pm \operatorname{SE}(n=4)$. One-way analysis of variance (ANOVA) was performed on all data and $P<0.05$ was considered statistically significant.

\section{Results}

\subsection{Sequence analysis of Rpdefs}

The 126 cDNA sequences of Rpdefs were deposited in GenBank under accession no. JX096678-JX096804. These sequences coded for defensins that fell in four defensin categories, which were named as Rpdef1, Rpdef2, Rpdef3 and Rpdef4, respectively. The complete coding sequence of Rpdef1, Rpdef2, Rpdef3 and Rpdef4 was of $219 \mathrm{bp}, 210 \mathrm{bp}, 207 \mathrm{bp}$ and $195 \mathrm{bp}$ in length, which encoded a polypeptide of 72, 69, 68 and 64 amino acids, respectively. The putative signal peptide of Rpdef1, Rpdef2 and Rpdef3 was identified at the $\mathrm{N}$-terminal sequence with the first 24 amino acids, while the putative signal peptide of Rpdef 4 comprised of the first 21 residues. The mature peptide of Rpdef1, Rpdef2, Rpdef3 and Rpdef4 consisted of 49, 46, 45 and 42 amino acids, which had a theoretical isoelectric point $(p \mathrm{I})$ of $6.86,8.23,8.50$ and 8.73 with an increasing predicted net charge of $0,+2,+3$ and +4 . The amino acid alignments indicated that Rpdef1 shared $46.9 \%$ identity with Rpdef2, 40.8\% with Rpdef3, and 34.7\% with Rpdef4. The transcript polymorphisms of these defensins have been shown in Supplemental figures.

\subsection{Structure prediction}

All of the Rpdefs have eight cysteine residues, which are predicted to form four disulfide bonds. However, the linkage patterns of the disulfide bridges predicted by different servers were not the same. Three-dimensional structure of Rpdef1 and Rpdef3 was predicted based on the template of Crassostrea gigas defensin (PDB ID: 2B68) with confidence $=99.9 \%$ (coverage $=86 \%$ ) and confidence $=92.1 \%$ (coverage $=84 \%$ ), respectively. However, 3-D structure of Rpdef2 and Rpdef4 was predicted based on the template of actinomycin (PDB ID: 2RU0) and scorpion toxin (PDB ID: 1SXM), with confidence $=63.3 \%$ (coverage $=57 \%$ ) and confidence $=83.0 \%$ (coverage $=55 \%$ ), respectively. All the predicted 3-D structures of Rpdefs possessed characteristic features of defensins, which comprised of one $\alpha$-helix and two antiparallel $\beta$ sheets (Fig. 2). Rpdef1 and Rpdef3 had similar speculated 3-D structures as $C$. gigas defensin, while Rpdef2 and Rpdef4 displayed unique 3-D structure respectively.

\section{Cgdef}

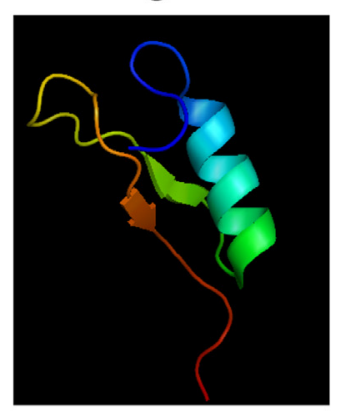

actinomycin
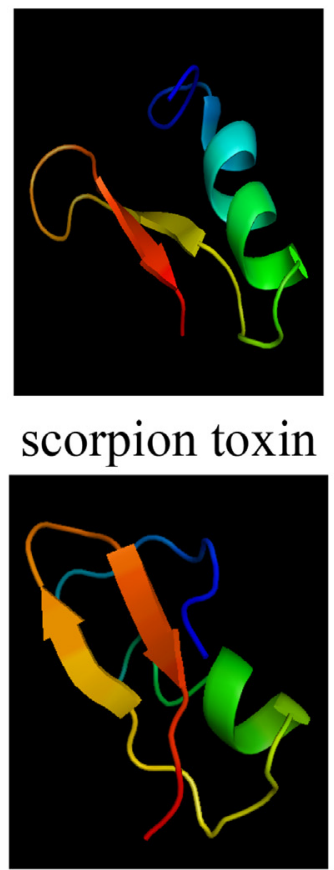

Rpdef1

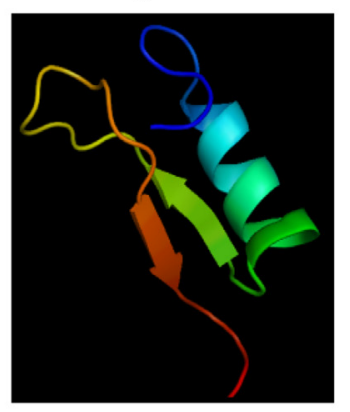

Rpdef2

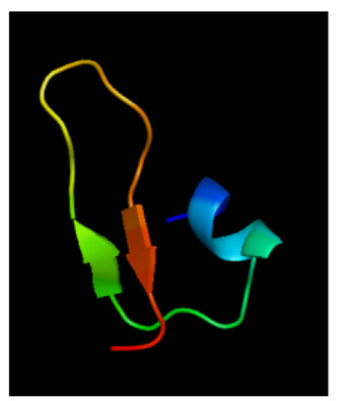

Rpdef4

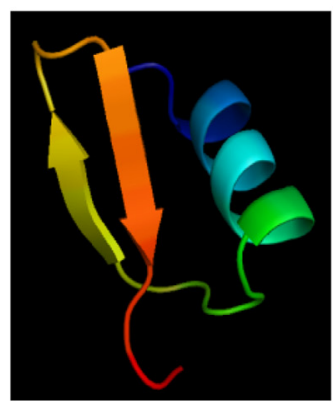

Fig. 2. Three-dimensional structures of Rpdefs and their counterparts. Cgdef (PDB ID: 2B68), actinomycin (PDB ID: 2RU0), scorpion toxin (PDB ID: 1SXM). 
Table 2

Sequences used for multiple alignments and phylogenetic analysis.

\begin{tabular}{lllll}
\hline Peptide & Species & MW Da & $p$ I & Accession numbers \\
\hline MGD1 & Mytilusgalloprovincialis & 4091 & 9.03 & P80571 \\
MGD2 & Mytilusgalloprovincialis & 4126 & 8.81 & AAD52660 \\
MedefA & Mytilusedulis & 4151 & 9.15 & P81610 \\
MedefB & Mytilusedulis & 4271 & 9.18 & P81611 \\
Cgdef & Crassostreagigas & 4642 & 8.73 & CAJ19280 \\
Cgdefh1 & Crassostreagigas & 4763 & 8.50 & ABD66301 \\
Cgdefh2 & Crassostreagigas & 4677 & 8.51 & ABD66302 \\
Cvdef & Crassostreavirginica & 4265 & 9.18 & P85008 \\
Hsdef & Hyriopsisschlegelii & 4877 & 7.78 & AEJ86348 \\
Hssdef & Haliotis discus discus & 4902 & 7.85 & ACZ15982 \\
Dpdef & Dreissenapolymorpha & 5684 & 6.27 & ACZ02692 \\
RpdefB & Ruditapesphilippinarum & 5287 & 7.79 & AEK78067 \\
MCdef & Ruditapesphilippinarum & 4975 & 8.71 & Adhya et al., 2012 \\
Rpdef1 & Ruditapesphilippinarum & 5435 & 6.86 & AFP50047 \\
Rpdef2 & Ruditapesphilippinarum & 5430 & 8.23 & AFP49990 \\
Rpdef3 & Ruditapesphilippinarum & 5330 & 8.50 & AFP49946 \\
Rpdef4 & Ruditapesphilippinarum & 4749 & 8.72 & AFP49977 \\
Hcdef1 & Hyriopsiscumingii & 4264 & 8.50 & Ren et al., 2011 \\
Hcdef2 & Hyriopsiscumingii & 4275 & 8.51 & Ren et al., 2011 \\
Hcdef3 & Hyriopsiscumingii & 4906 & 8.32 & Ren et al., 2011 \\
Hcdef4 & Hyriopsiscumingii & 4880 & 8.66 & Ren et al., 2011 \\
Hcdef5 & Hyriopsiscumingii & 7112 & 8.48 & Ren et al., 2011 \\
Hcdef6 & Hyriopsiscumingii & 4602 & 8.50 & Ren et al., 2011 \\
\hline & & & & \\
\hline & & & & \\
& & & &
\end{tabular}

\subsection{Multiple alignment and phylogenetic relationships}

The sequences used for multiple alignments and phylogenetic analysis were shown in Table 2. Multiple alignments indicated that eight cysteine residues and C-terminal motif (-RRSIQ-) were highly conserved in Rpdefs (Fig. 3a), whereas only four cysteine residues were conserved in mollusk defensins (Fig. 3b). Rpdefs had eight cysteine residues like defensins from the Manila clam (McDef), pacific oyster (Cgdef, Cgdefh1 and Cgdefh2), Mediterranean mussel (MGD1, MGD2) and triangle-shell pearl mussel (Hcdef5, Hcdef6), while some mollusk defensins contain a pattern of six conserved cysteine residues (Fig. 3b).
The phylogeny tree was generated using the models GTR $+\mathrm{G}$ for mollusk defensins determined by the Akaike information criterion. Phylogenetic analysis showed that Rpdef2 and Rpdef3 first clustered together, then segregated with Rpdef1, Rpdef4 and McDef successively and formed as a subclade (Fig. 4). The subclade of defensins from Manila clam first rooted with defensins from fresh water mussels Hyriopsis schlegelii and Hyriopsis cumingii, further clustered with defensins from oysters and mussels and formed a clade with RpdefB (a defensin deposited in the Genbank database) from the Manila clam. At last, these defensins grouped with defensins from the abalone and zebra mussel. These results indicated that RpdefB diverged from Rpdefs and McDef before the divergence of Manila clam with pacific oyster and Mediterranean mussel.

\section{4. mRNA polymorphism and diversity of Rpdefs}

A total of 126 clones were sequenced and virtually translated into amino acid sequences. Rpdef 3 accounted for $60 \%$ frequency of occurrence of the clones, with Rpdef1, Rpdef 2 and Rpdef 4 accounting for $17 \%, 13 \%$ and $10 \%$ of the clones (Table 3 ). Multiple alignments indicated that Rpdef 1 had 8 different kinds of amino acid sequences of the 22 sequences, as for Rpdef 2 with 4 out of 17 sequences, Rpdef 3 with 16 out of 75 sequences and Rpdef 4 with 5 out of 12 sequences. The above results suggested that the transcript variation of Rpdef1 and Rpdef4 was larger than that of Rpdef2 and Rpdef3. The phylogeny trees were also constructed to compare the sequence variance between these four Rpdef genes with PhyML 3.0. The models employed for phylogeny analyses (HKY $+\mathrm{G}$ for Rpdef1, HKY $+\mathrm{G}+\mathrm{I}$ for Rpdef2, GTR for Rpdef3, and $\mathrm{HKY}+\mathrm{I}$ for Rpdef4) were determined by the Akaike information criterion. The phylogeny analysis indicated that the Rpdef1 sequences are more divergent than those of Rpdef2, Rpdef3 and Rpdef4 (Fig. 5).

With regard to clams from Dalian, frequencies of occurrence were extremely different, with Rpdef3 accounting for $82 \%$ of the

a

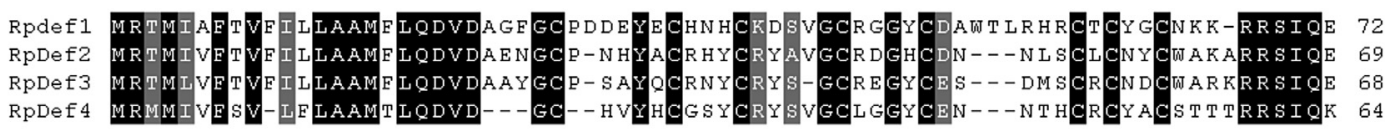

b

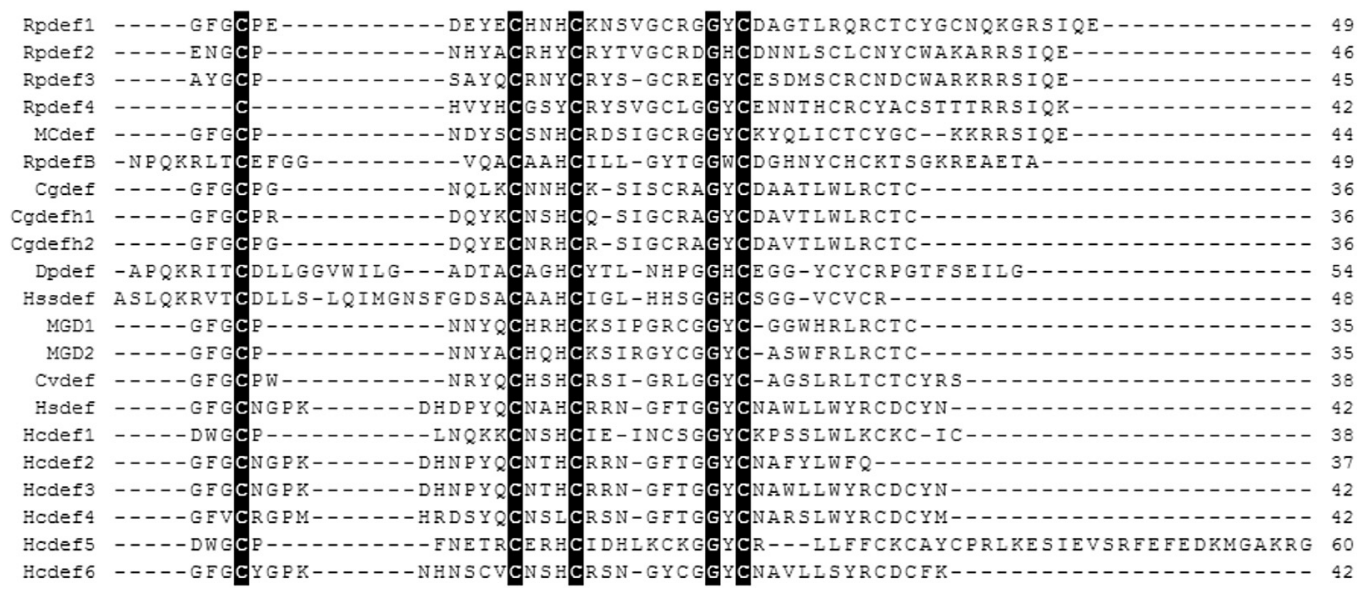

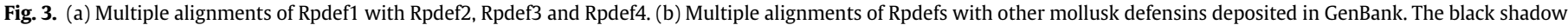

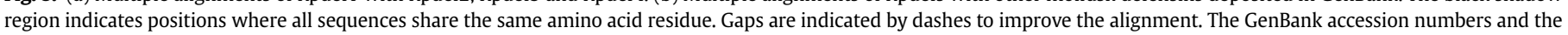
species are shown in Table 2. 


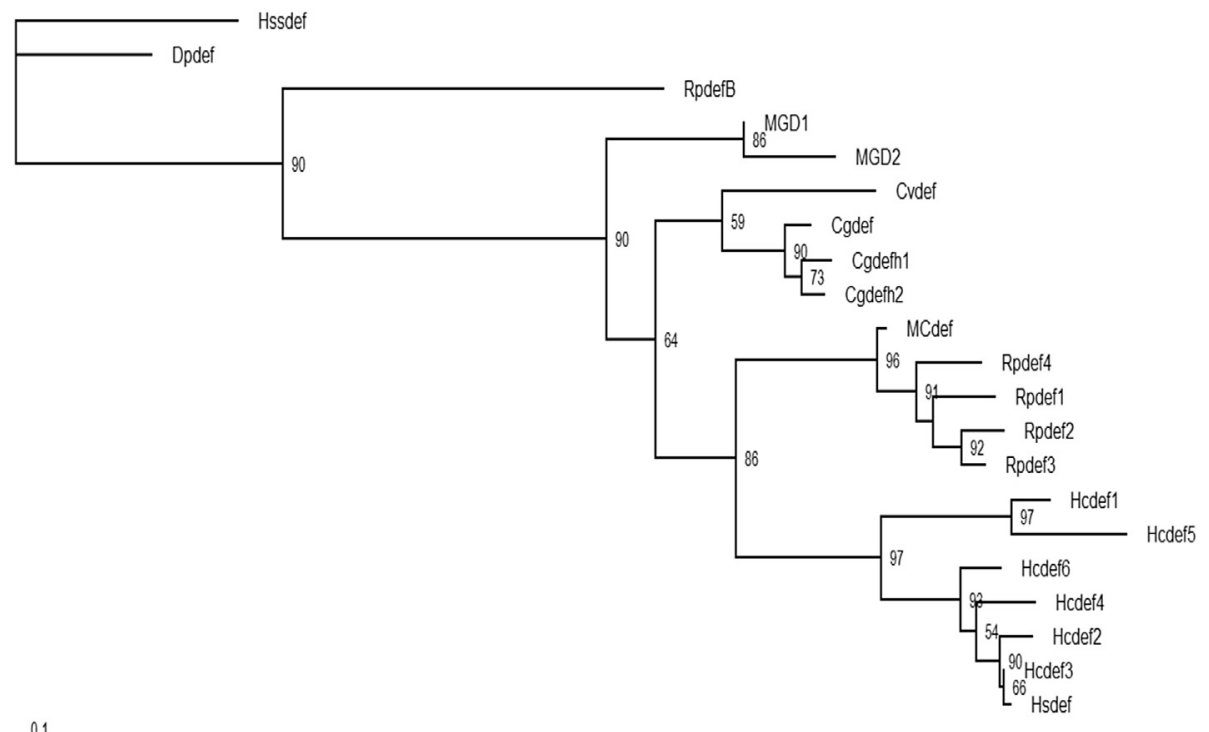

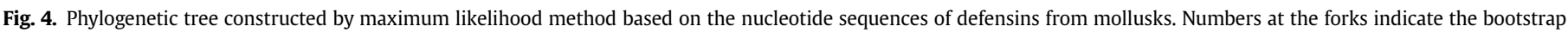
values (in \%) out of 100 replicates. The sequences used to construct phylogeny trees of defensins are shown in Table 2.

clones and Rpdef4 present in only one clone. Other coding sequences have been found in 3-5 clones. However, as for clams from Qingdao and Hangzhou, Rpdef3 accounted for $43 \%$ and $48 \%$ of the clones; Rpdef2 and Rpdef4 showed the least frequency of occurrence (Table 3).

As concerned to tissue distribution of the different Rpdef transcripts, 52 clones were present in gills, whereas 36 and 38 clones were present in hemocytes and digestive gland respectively. As for Rpdef1, 50\% clones were present in hemocytes, while for Rpdef2 and Rpdef3, the clones were almost averagely present in three tissues. The clones of Rpdef 4 were predominantly present in gills and digestive gland (Table 3 ).

\subsection{Tissue-specific expressions profiles of Rpdefs mRNAs}

The tissue distribution of Repdef mRNAs was investigated by qRT-PCR with $\beta$-actin as internal control. During the qRT-PCR assays, only one peak was detected at the corresponding melting temperature in the dissociation curve analysis, suggesting that the PCR was specifically amplified. The transcripts of Rpdefs were detected in all the tissues examined, including gills, digestive gland, hemocytes, mantle and foot. The transcripts of Rpdefs with the exception of Rpdef 4 were dominantly expressed in hemocytes, moderately expressed in gills, mantle and foot (Fig. 6A, B, C), and least detected in digestive gland. However, the expression level of Rpdef4 mRNA was high in gills and hemocytes, moderate in mantle and digestive gland, and least in foot (Fig. 6D).

Table 3

Number of clones of Rpdefs in different location and different tissues.

\begin{tabular}{|c|c|c|c|c|c|c|}
\hline \multirow[t]{3}{*}{ Genes } & \multicolumn{6}{|c|}{ Number of clones } \\
\hline & \multicolumn{3}{|c|}{ Location } & \multicolumn{3}{|l|}{ Tissue } \\
\hline & Dalian & Qingdao & Hangzhou & Hemcoytes & Gill & Digestive gland \\
\hline Rpdef1 & 5 & 11 & 6 & 11 & 8 & 3 \\
\hline Rpdef2 & 3 & 6 & 8 & 6 & 6 & 5 \\
\hline Rpdef3 & 40 & 20 & 15 & 18 & 32 & 25 \\
\hline Rpdef4 & 1 & 9 & 2 & 1 & 6 & 5 \\
\hline
\end{tabular}

3.6. Temporal expression profiles of Repdefs mRNAs in hemocytes post bacterial challenge

Following bacterial challenge, the expression levels of all Rpdefs in hemocytes (Fig. 7) increased significantly at $12 \mathrm{~h}$ post challenge $(P<0.05)$. For Rpdef1, the expression level returned to the original level at $24 \mathrm{~h}$ and $48 \mathrm{~h}$ post challenge (Fig. 7A). However, the transcripts of Rpdef2 and Rpdef3 were significantly inhibited at $24 \mathrm{~h}$ and $48 \mathrm{~h}$ following challenge $(P<0.05)$ (Fig. $7 \mathrm{~B}, \mathrm{C})$. The expression level of Rpdef 4 was significantly up-regulated at $12 \mathrm{~h}$ and $24 \mathrm{~h}$, and down-regulated at $48 \mathrm{~h}$ post challenge $(P<0.05)$ (Fig. 7D).

\subsection{Evolutionary analysis of Rpdefs and other mollusk defensin genes}

Phylogeny-based codon substitution models were used to identify the codons under positive selection. For all Rpdefs, the M0-M3 comparison revealed that M3 was not a better fit to the data than $\mathrm{M0}(2 \Delta \mathrm{I}=130 ; P>0.05)$. The comparison of null models (M1a and M7) with their corresponding alternative models (M2a and M8) rejected the null models (Table 4), thus indicated that the variants of Rpdefs have evolved under positive selection with the positively selected sites $R^{63}$ and $R^{64}$ falling in the C-terminus. For Rpdef1, Rpdef2 and Rpdef4, the comparison of null models with their corresponding alternative models agreed the null models $(P$ $>0.05$ ), suggesting no amino acid residue under positive selection. However, the amino acid residues $R^{63}, R^{64}, S^{65}, I^{66}$ and $Q^{67}$ in Rpdef3 were detected under positive selection (Table 5).

The M1-M0 comparison revealed that model M1 was better fit to the data than M0 $(P<0.05)$, indicating the $\omega$ ratios were different among lineages of the phylogeny tree. The lineagespecific selection test showed that the $\omega$ values along most examined lineages ( 28 out of 39 ) were less than 1 , whereas there were also some branches and internal branches with high $\omega$ values (Table 6). As shown in Fig. 6, the Rpdef3 lineage and Rpdefs branch (formed by Rpdef1, Rpdef2, Rpdef3 and Rpdef4) had $\omega$ ratios $>1$, indicating Rpdef3 lineage and Rpdefs branch undergo positive selection. In the clade of oysters, the $\omega$ values for the Cgdefh2 lineage and Cgdefs branch (formed with Cgdefh1 and Cgdefh2) were more than 1 . There were also three lineages with 
Rpdef1

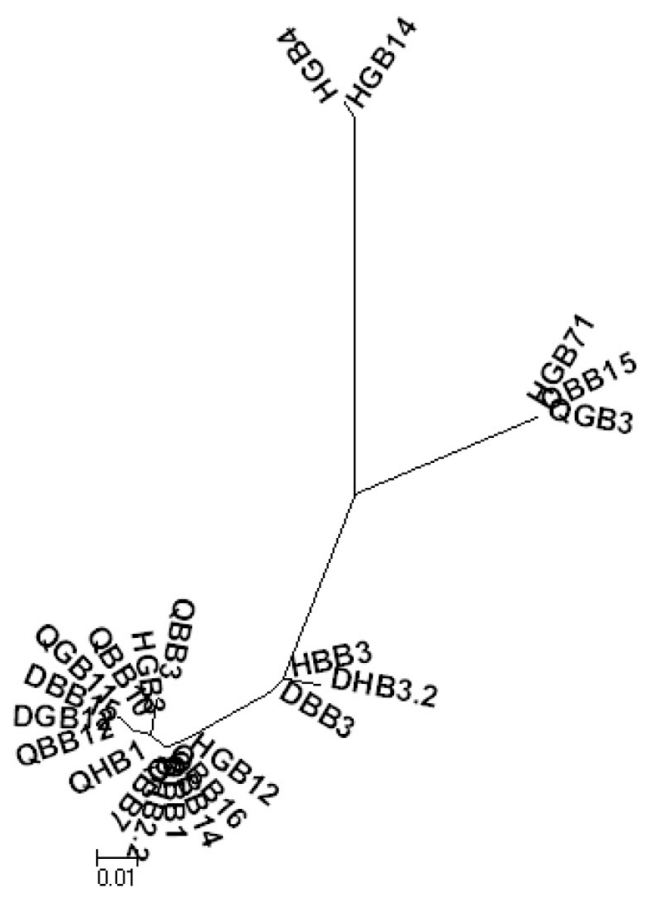

Rpdef3

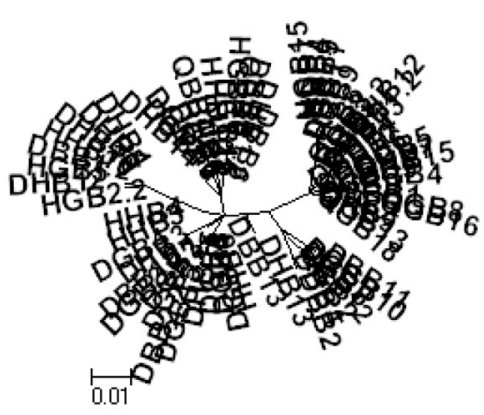

Rpdef2

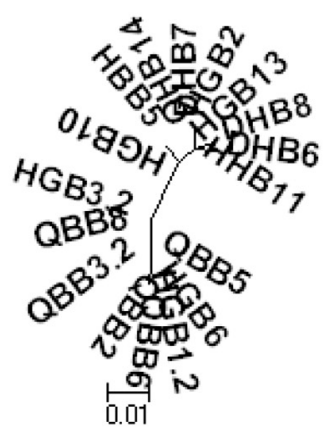

Rpdef4

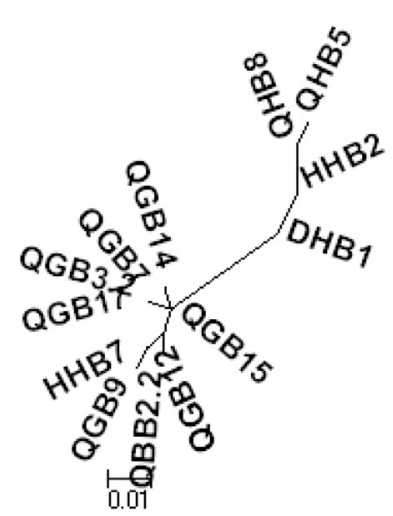

Fig. 5. Phylogenetic tree constructed by maximum likelihood method based on the nucleotide sequences of Rpdefs.

very high $\omega$ ratios in the clade of fresh water pearl mussel, implying positive selection on these lineages. However, the $\omega$ ratio value for the Mediterranean mussel branch was $<1$, implying no positive selection on this branch. The Manila clam branch (without RpdefB) had high $\omega$ ratios $>1$, revealing that this branch is under positive selection pressure during evolution (Fig. 8). In addition, there were also some clades from different taxon exhibited high $\omega$ ratios. The site-specific models were used to test for heterogeneous selective pressure at amino acid sites. The M1a-M2a comparison revealed that M2a was better fit to the data $(P<0.01)$. LRTs also gave significantly better results for M8 $(P<0.01)$. Under the M2a model, 23 amino acids within the mature peptide regions are under positive selection, while 17 amino acid residues are positively selected under the M8 model. We considered a site under positive selection if the BEB posterior probability is $>0.95$. Under M2a and M8 model, 14 and 6 positively selected sites were detected with BEB posterior probability $>0.95$ respectively (Table 6 ).

\section{Discussion}

Marine mollusks account for a large quantity of current global aquaculture output. With the rapid development of intensive mariculture, some cultured species have been seriously affected by diseases and mortalities in recent years $[39,40]$. Therefore, basic knowledge on the innate immunity of commercially important mollusks is urgently needed, especially characterization of the immune-associated molecules and their functions. AMPs constitute an important first-line defense of the immune system in mollusks. Among these naturally occurring antibiotic peptides, defensins form a unique family of cysteine-rich cationic and structured polypeptides, serving as effector molecules of innate immunity [41]. Although Mollusca are the largest and most diverse phylum of animals next to arthropods, AMPs such as defensins have been characterized only in a few farmed species, such as oysters, mussels and abalones. Therefore, there is still a great potential to unveil new AMP molecules in this phylum [20]. 

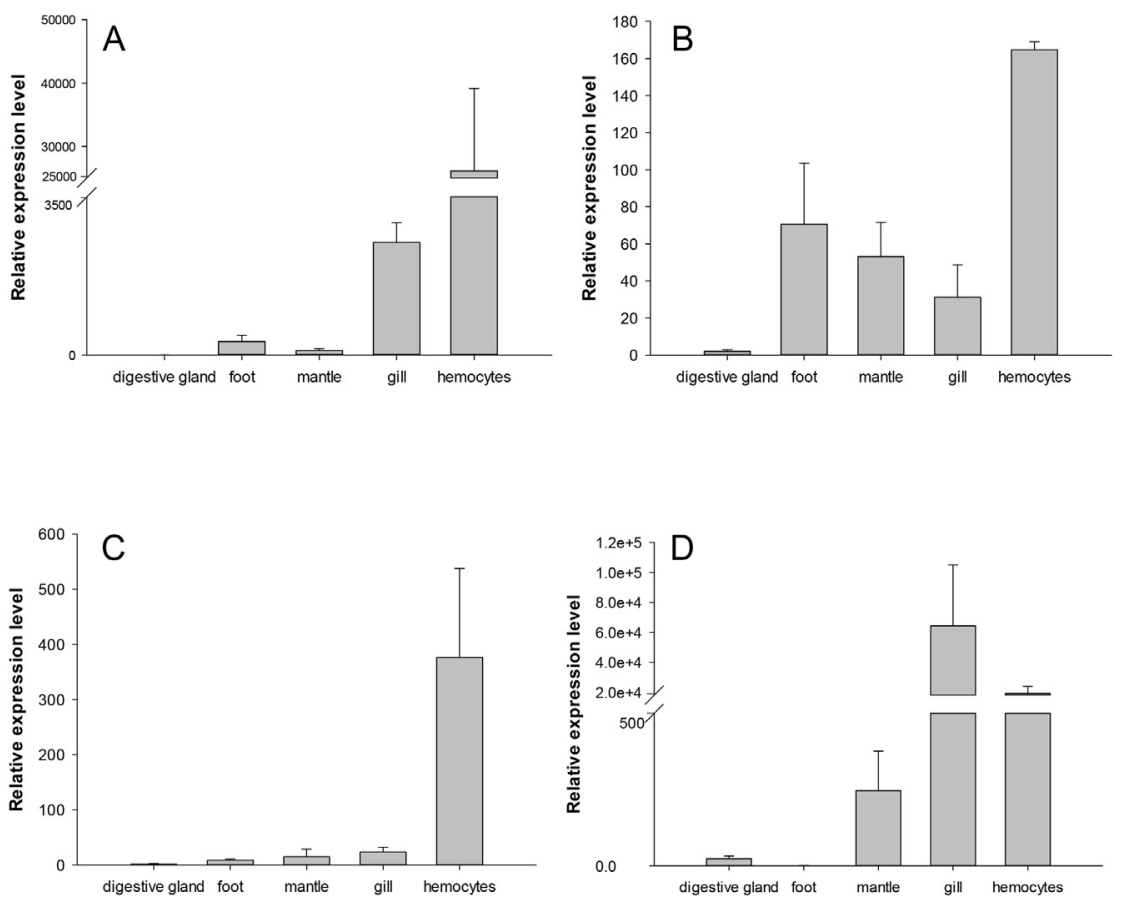

Fig. 6. Tissue-specific expression profiles of Rpdefs mRNAs measured by qRT-PCR. The mRNA expression level is calculated relative to $\beta$-actin expression. Each symbol and vertical bar represents the mean $\pm \mathrm{SE}(\mathrm{n}=4)$. A-Rpdef1, B-Rpdef2, C-Rpdef3, D-Rpdef4.
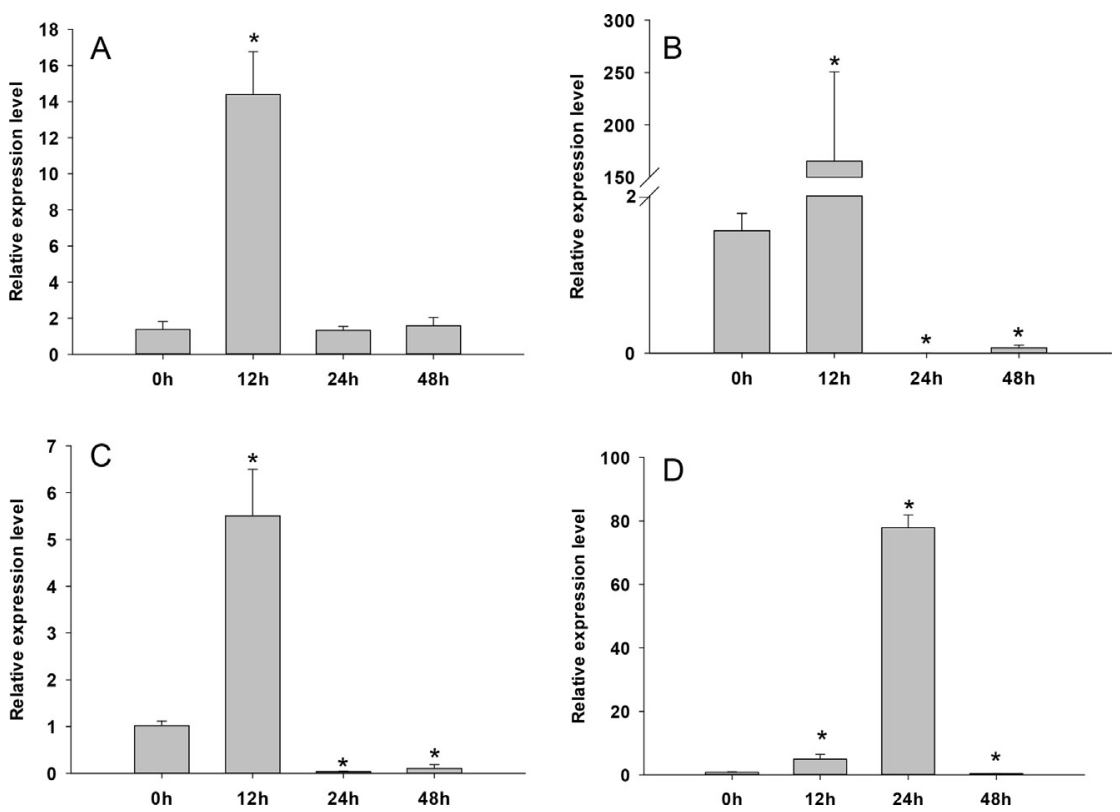

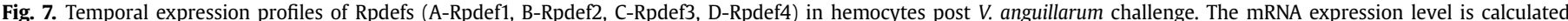
relative to $\beta$-actin expression. Each symbol and vertical bar represents the mean $\pm \mathrm{SE}(\mathrm{n}=4)$. Significant difference from control is indicated with an asterisk at $P<0.05$.

Table 4

Comparison among different nested models to test for positive selection among codons of all Rpdef sequences.

\begin{tabular}{|c|c|c|c|c|}
\hline Model & $\ln L$ & Estimates of parameters & $2 \Delta \mathrm{I}$ & Positively selected sites \\
\hline M1a (nearly neutral) & -1702 & $\mathrm{P} 0=0.25618,(\mathrm{p} 1=0.74382)$ & 46 & Not allowed \\
\hline M2a (positively selection) & -1679 & $\mathrm{p} 0=0.22939, \mathrm{p} 1=0.73902,(\mathrm{p} 2=0.03159), \omega 0=0.08282,(\omega 1=1), \omega 2=14.61681$ & $P<0.01$ & $63 R^{*}, 64 R^{*}$ \\
\hline M7 (beta) & -1703 & $\mathrm{p}=0.43916, \mathrm{q}=0.33694$ & 46 & Not allowed \\
\hline M8 (beta \& w > 1) & -1680 & $\mathrm{p} 0=0.96839,(\mathrm{p} 1=0.03161), \mathrm{p}=0.14181, \mathrm{q}=0.04183, \omega \mathrm{s}=14.21207$ & $P<0.01$ & $63 R^{*}, 64 R^{*}$ \\
\hline
\end{tabular}


Table 5

Comparison among different nested models to test for positive selection among codons of Rpdef1, Rpdef2, Rpdef3 and Rpdef4.

\begin{tabular}{|c|c|c|c|c|c|}
\hline & Model & $\ln L$ & Estimates of parameters & $2 \Delta \mathrm{I}$ & Positively selected sites \\
\hline \multirow[t]{4}{*}{ Rpdef1 } & M1a (nearly neutral) & -700.29 & $\mathrm{p} 0=0.25658,(\mathrm{p} 1=0.74342)$ & 4.62 & Not allowed \\
\hline & $\begin{array}{l}\text { M2a (positively } \\
\text { selection) }\end{array}$ & -697.98 & $\begin{array}{l}\mathrm{p} 0=0.08753, \mathrm{p} 1=0.69312,(\mathrm{p} 2=0.21935), \omega 0=0.00000 \\
(\omega 1=1), \omega 2=7.59516\end{array}$ & $P>0.05$ & $\begin{array}{l}\text { 4M, 10F, 28P, 29D, 32Y, 40D, 50D, 51A, } \\
52 \mathrm{~W}, 53 \mathrm{~T}, 55 \mathrm{R}, 56 \mathrm{H}, 65 \mathrm{~K}, 67 \mathrm{R}\end{array}$ \\
\hline & M7 (beta) & -700.34 & $\mathrm{p}=0.14734, \mathrm{q}=0.04084$ & 4.82 & Not allowed \\
\hline & M8 (beta \& $\mathrm{w}>1$ ) & -697.93 & $\begin{array}{l}\mathrm{p} 0=0.78255,(\mathrm{p} 1=0.21745), \mathrm{p}=0.10482, \mathrm{q}=0.02625 \\
\omega \mathrm{s}=6.67735\end{array}$ & $P>0.05$ & $\begin{array}{l}\text { 4M, 10F, 28P, 29D*, 30D, 32Y, 40D, 50D, } \\
51 \mathrm{~A}, 52 \mathrm{~W}, 53 \mathrm{~T}, 55 \mathrm{R}, 56 \mathrm{H}, 65 \mathrm{~K}, 67 \mathrm{R}\end{array}$ \\
\hline \multirow[t]{4}{*}{ Rpdef2 } & M1a (nearly neutral) & -319.19 & $\mathrm{p} 0=0.00001,(\mathrm{p} 1=0.99999)$ & 5.00 & Not allowed \\
\hline & $\begin{array}{l}\text { M2a (positively } \\
\text { selection) }\end{array}$ & -316.69 & $\begin{array}{l}\mathrm{p} 0=0.00000, \mathrm{p} 1=0.67091,(\mathrm{p} 2=0.32909), \omega 0=1.00000 \\
(\omega 1=1), \omega 2=999.00000\end{array}$ & $P>0.05$ & all amino acids \\
\hline & M7 (beta) & -319.19 & $\mathrm{p}=2.02546, \mathrm{q}=0.00500$ & 5.00 & Not allowed \\
\hline & M8 (beta \& w > 1) & -316.69 & $\begin{array}{l}\mathrm{p} 0=0.66973,(\mathrm{p} 1=0.3302), \mathrm{p}=0.00500, \mathrm{q}=1.57842 \\
\omega \mathrm{s}=999.00000\end{array}$ & $P>0.05$ & all amino acids \\
\hline \multirow[t]{4}{*}{ Rpdef3 } & M1a (nearly neutral) & -733.46 & $\mathrm{p} 0=0.78832,(\mathrm{p} 1=0.21168)$ & 63.74 & Not allowed \\
\hline & $\begin{array}{l}\text { M2a (positively } \\
\text { selection) }\end{array}$ & -701.59 & $\begin{array}{l}\mathrm{p} 0=0.56707, \mathrm{p} 1=0.35845,(\mathrm{p} 2=0.07449), \omega 0=0.07666 \\
(\omega 1=1), \omega 2=21.89229\end{array}$ & $P<0.01$ & 63R* $64 \mathrm{R}^{*}, 65 \mathrm{~S}^{*}, 66 \mathrm{I}^{*}, 67 \mathrm{Q}^{*}$ \\
\hline & M7 (beta) & -734.99 & $\mathrm{p}=0.05393, \mathrm{q}=0.06892$ & 66.66 & Not allowed \\
\hline & M8 (beta \& $\mathrm{w}>1$ ) & -701.69 & $\begin{array}{l}\mathrm{p} 0=0.92554,(\mathrm{p} 1=0.07446), \mathrm{p}=0.06396, \mathrm{q}=0.07578 \\
\omega \mathrm{s}=20.39602\end{array}$ & $P<0.01$ & 63R* $\mathrm{R}^{*}, 64 \mathrm{R}^{*}, 65 \mathrm{~S}^{*}, 66 \mathrm{I}^{*}, 67 \mathrm{Q}^{*}$ \\
\hline \multirow[t]{4}{*}{ Rpdef4 } & M1a (nearly neutral) & -320.09 & $\mathrm{p} 0=0.00001,(\mathrm{p} 1=0.99999)$ & 0.50 & Not allowed \\
\hline & $\begin{array}{l}\text { M2a (positively } \\
\text { selection) }\end{array}$ & -319.84 & $\begin{array}{l}\mathrm{p} 0=0.40603, \mathrm{p} 1=0.00000,(\mathrm{p} 2=0.59397), \omega 0=0.00000 \\
(\omega 1=1), \omega 2=2.45542\end{array}$ & $P>0.05$ & $14 \mathrm{~A}, 19 \mathrm{D}, 46 \mathrm{~N}$ \\
\hline & M7 (beta) & -320.09 & $\mathrm{p}=1.18224, \mathrm{q}=0.00500$ & 0.50 & Not allowed \\
\hline & M8 (beta \& $\mathrm{w}>1$ ) & -319.84 & $\begin{array}{l}\mathrm{p} 0=0.40603,(\mathrm{p} 1=0.59397), \mathrm{p}=0.00845, \mathrm{q}=1.78436 \\
\omega \mathrm{s}=2.45543\end{array}$ & $P>0.05$ & $14 \mathrm{~A}, 19 \mathrm{D}, 24 \mathrm{H}, 26 \mathrm{Y}, 27 \mathrm{H}, 34 \mathrm{Y}, 42 \mathrm{Y}, 46 \mathrm{~N}$ \\
\hline
\end{tabular}

Table 6

Parameter estimates and log-likelihood values under different models of variable $\omega$ ratios among sites. Site numbers and amino acids refer to the Rpdef1 sequence.

\begin{tabular}{|c|c|c|c|c|c|c|}
\hline Model & Model code & $\ln L$ & Estimates of parameters & $2 \Delta l$ & $P$ Value & Positively selected sites \\
\hline \multirow[t]{2}{*}{$\begin{array}{l}\text { Branch } \\
\text { model }\end{array}$} & One ratio model & -3427.1 & $\omega=0.39891$ & $\begin{array}{l}57.2 \\
(\mathrm{df}=38)\end{array}$ & $P<0.05$ & NA \\
\hline & $\begin{array}{l}\text { Free-ratio } \\
\text { model }\end{array}$ & -3398.5 & $\begin{array}{l}\omega \text { estimated independently for each } \\
\text { branch (see Fig. 4) }\end{array}$ & & & \\
\hline \multirow[t]{4}{*}{ Site model } & M1a & -3426.9 & $\mathrm{P} 0=0.01754(\mathrm{p} 1=0.98246)$ & 51.2 & $P<0.01$ & Not allowed \\
\hline & M2a & -3401.3 & $\begin{array}{l}\mathrm{p} 0=0.01756, \mathrm{p} 1=0.63873 \\
(\mathrm{p} 2=0.34371), \omega 0=0.00000 \\
(\omega 1=1), \omega 2=4.82950\end{array}$ & & & $\begin{array}{l}28 \mathrm{P}^{*}, 31 \mathrm{E}^{*}, 32 \mathrm{Y}^{* *}, 33 \mathrm{E}, 34 \mathrm{C}, 35 \mathrm{H}^{* *}, 37 \mathrm{H}, 38 \mathrm{C}^{*}, 40 \mathrm{D}^{* *}, 41 \mathrm{~S}, \\
43 \mathrm{G}, 44 \mathrm{C}, 47 \mathrm{G}, 48 \mathrm{Y}^{* *}, 49 \mathrm{C}^{*}, 50 \mathrm{D}, 51 \mathrm{~A}^{* *}, 52 \mathrm{~W}^{*}, 53 \mathrm{~T}^{* *}, 54 \mathrm{~L}^{* *}, \\
55 \mathrm{R}^{* *}, 56 \mathrm{H}^{* *}, 57 \mathrm{R}\end{array}$ \\
\hline & M7 & -3363.9 & $\mathrm{p}=0.06961, \mathrm{q}=0.09195$ & 126 & $P<0.01$ & Not allowed \\
\hline & M8 & -3426.9 & $\begin{array}{l}\mathrm{p} 0=0.01754, \mathrm{p}=0.00500 \\
\mathrm{q}=0.00500,(\mathrm{p} 1=0.00500) \\
\mathrm{w}=1.00000\end{array}$ & & & $\begin{array}{l}28 \mathrm{P}, 31 \mathrm{E}, 32 \mathrm{Y}^{* *}, 33 \mathrm{E}, 35 \mathrm{H}^{* *}, 38 \mathrm{C}, 40 \mathrm{D}^{*}, 43 \mathrm{G}, 48 \mathrm{Y}, 49 \mathrm{C}, 50 \mathrm{D} \\
51 \mathrm{~A}^{* *}, 52 \mathrm{~W}, 53 \mathrm{~T}, 54 \mathrm{~L}^{* *} 55 \mathrm{R}, 56 \mathrm{H}^{* *}\end{array}$ \\
\hline
\end{tabular}

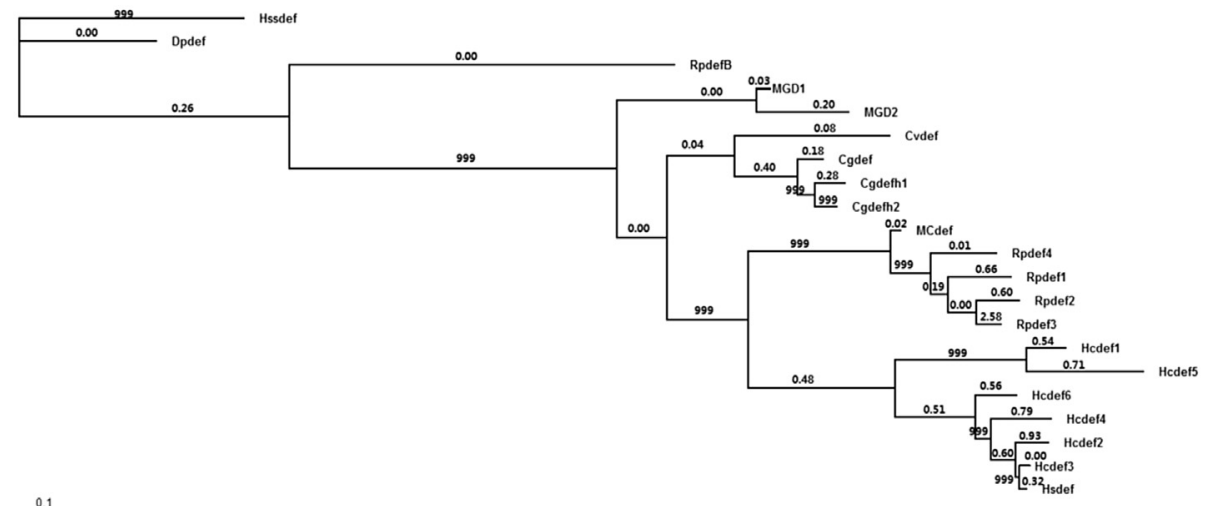

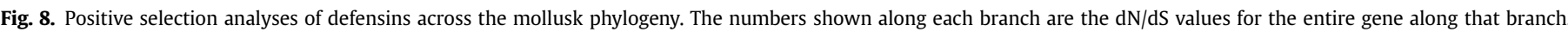

In this study, we have characterized four types of defensins from the commercially important Manila clam. Presently, six defensin isoforms including McDef and RpdefB (deposited in the Genbank database) have been isolated from this species. The amino acid alignments indicated that Rpdef1 shared 65.3\% with McDef, 46.9\% identity with Rpdef2, 40.8\% with Rpdef3, and 34.7\% with Rpdef4, whereas it had only $18.4 \%$ identity with RpdefB. Like other mollusk defensins, all of these defensins from Manila clam with the exception of Rpdef1 had a net positive charge, and their theoretical pIs were more than 8.0 (Table 2). However, Rpdef1 and defensin from Dreissena 
polymorpha (Dpdef) had a theoretical $\mathrm{pI}$ of less than 7 , and possessed a net charge of 0 and -1 , respectively. Although the vast majority of AMPs are cationic in nature, a significant number of anionic AMPs have also been reported. As for the non-cationic defensins, perhaps they can dock to the bacterial membrane via the positively charged and hydrophobic loop near their C-terminus [42].

Rpdef3 sequences accounted for the most percentage of the Rpdefs sequences, suggesting this defensin might play a more important role than other Rpdefs in this clam. The occurrence frequencies of different Rpdefs varied in clams from different geographical locations. The transcript variation was perhaps related to pathogen load in clams and other possible environmental factors of different sites, which may have forced the defensin genes to diversify [43]. The transcript sequences of Rpdef2 and Rpdef3 were almost averagely present in three tissues, while the transcript sequences of Rpdef1 were mainly present in hemocytes and Rpdef4 were mainly detected in gills and digestive gland. These above results indicated different Rpdefs perhaps played different immune functions in specific tissues. In addition, multiple alignment and phylogeny analysis showed that Rpdef1 had a higher diversity than other Rpdefs, while Rpdef3 had the lowest diversity. The divergence of Rpdef1 was likely due to purifying selection acting in a long term manner, while the polymorphism reduction of Rpdef3 was probably caused by a recent selective pressure [26], which has been detected by evolutionary analysis in the present study.

In this study, the constitutive expression of all Rpdefs was mainly detected in hemocytes, which are primarily responsible for the defense against pathogens in bivalves. Similarly, it has been reported that oyster defensin (Cgdefh2) and mussel defensins (MGD1 and MGD2) were abundantly expressed in hemocytes $[14,16,21]$. As for Rpdef4, the highest expression was found in gills, which is continuously exposed to environmental stress factors such as toxic substances and pathogens. These types of tissue-distribution profiles suggested these defensins could respond promptly to bacterial challenge. However, it was reported that MCdef transcript was expressed at the highest level in adductor muscle of the Manila clam [32]; and the abalone defensin transcript was highly expressed in the mantle and hepatopancreas [19]. Thus, these results demonstrated that these defensins may play different immune roles in different tissues of these bivalves.

In order to further understand the possible biological functions of the Rpdefs, their mRNA expression patterns post bacterial challenge were examined at different time intervals in hemocytes. The expression of all Rpdefs was up-regulated remarkably after $12 \mathrm{~h}$ bacterial challenge compared to that of the control, indicating that the transcripts of Rpdefs were induced to fight against the bacteria. The up-regulation of defensin transcripts was also observed in disk abalone [19], Manila clam (MCdef) [32] and freshwater pearl mussel Hyriopsis schlegelii [44]. Then the expression levels of all Rpdefs except Rpdef4 returned to original level or were inhibited at $24 \mathrm{~h}$ and $48 \mathrm{~h}$ post challenge. The possible reason of downregulation was that there was no need to produce large amount of defensins with the progressive clearance of the invasive bacteria. In fact, the expression of oyster defensin (Cgdefh2) and mussel defensin (MGD2) was also found to be significantly down-regulated in hemocytes after bacterial infection $[14,16]$. Above all, the transcriptional up-regulation of Rpdefs against bacterial challenge as well as their highly constitutive expression in hemocytes indicated that they may play important roles in innate immunity.

It has been shown that genes involved in the immune system of various animals typically show a faster rate of amino acid substitutions and have evolved under positive selection $[45,46]$. Due to their direct interaction with altered/new pathogens, AMPs exhibit an extraordinary diversity in their biochemical and biological functions. It has been reported that positive Darwinian selection was the major driving force in the generation of diverse AMPs [47].

Consistent with earlier studies on molecular evolution of several AMPs [25-31], our results suggested that all Rpdefs evolved through positive selection. Positively selected codons $R^{63}$ and $R^{64}$ had been detected in the $\mathrm{C}$-terminal regions of all these Rpdefs. In addition, the amino acid residues $S^{65}, I^{66}$ and $Q^{67}$ in the C-terminal region of Rpdef3 were also detected under positive selection. Similarly, the majority of positively selected sites are located in the C-terminal region of myticin-C $[25,48]$. Based on the predicted tertiary structure of Rpdefs, the positively selected amino acids fall in the predicted C-terminal coils (data not shown), which are highly exposed at the surface of Rpdefs. Moreover, the positively charged $\mathrm{R}^{63}$ and $\mathrm{R}^{64}$ perhaps increase the binding ability of peptide to the bacteria. It had been demonstrated that positively charged amino acids on the surface of the defensin could greatly improve their antibacterial activity, probably by promoting a better binding to the cell wall or membrane of target bacteria [49,50]. Therefore, the positively selected amino acids might have a functional relevance by modifying the charge distribution of Rpdefs. Further investigation on the functional roles of C-terminal domain in defensins will be crucial to interpret these observations [48].

The results of the present study provide strong evidence that positive selection is the major driving force in generating high diversity in Rpdefs. Zhu et al. [47] also reported that many members of the CS- $\alpha \beta$ superfamily exhibited molecular diversity and diverse biological functions, and suggested that positive Darwinian selection is the major driving force in generating such diversity. The direct involvement of these peptides with the altered pathogens in a changing environment is probably the cause of such adaptive molecular evolution of these AMPs [25].

\section{Conclusions}

We characterized the complete coding sequences and predicted the 3-D structures of four defensins from the Manila clam. Next, we studied the diversity of defensins from three geographical clam populations and found that Rpdef3 accounted for about $60 \%$ frequency of Rpdefs occurrence in these clams. The expression profiles post bacterial challenge suggested that Rpdefs were involved in the host defense in this clam. Furthermore, it was found that Rpdef3 and all Rpdefs were under positive selection with positively selected amino acid residues detected in the C-terminal regions, which perhaps have a functional relevance by modifying the charge distribution of Rpdefs. Therefore, positive selection could be the major driving force in generating high diversity of defensins in the Manila clam.

\section{Acknowledgements}

We thank Dr. Xiutang Yuan, Dr. Tianlong Qiu and Dr. Changkao $\mathrm{Mu}$ for their help in sample collection. This research was supported by grants from NSFC (Grant No. 41476126), the Strategic Priority Research Program of the Chinese Academy of Science (Grant No. XDA11020405) and Key Research Program of the Chinese Academy of Sciences (Grant No. KZZD-EW-14).

\section{Appendix A. Supplementary data}

Supplementary data related to this article can be found at http:// dx.doi.org/10.1016/j.fsi.2015.09.008

\section{References}

[1] K. Sőderhäll, Invertebrate immunity, Dev. Comp. Immunol. 23 (1999) 263-266. 
[2] E.S. Loker, C.M. Adema, S.M. Zhang, T.B. Kepler, Invertebrate immune systemsnot homogeneous, not simple, not well understood, Immunol. Rev. 198 (2004) $10-24$.

[3] M. Zasloff, Antimicrobial peptides of multicellular organisms, Nature 415 (2002) 389-395.

[4] L. Song, L. Wang, L. Qui, H. Zhang, Bivalve Immunity, in: K. Soderhall (Ed.), Advances in Experimental Medicine and Biology - Invertebrate Immunity, Springer, New York, USA, 2010, p. 708, 44-65.

[5] A.J. Otero-González, B.S. Magalhães, M. Garcia-Villarino, C. López-Abarrategui, D.A. Sousa, S.C. Dias, et al., Antimicrobial peptides from marine invertebrates as a new frontier for microbial infection control, FASEB J. 24 (2010) $1320-1334$.

[6] M. Charlet, S. Chernysh, H. Philippe, C. Hetru, J.A. Hoffmann, P. Bulet, Innate immunity. Isolation of several cysteine-rich antimicrobial peptides from the blood of a mollusc, Mytilus edulis, J. Biol. Chem. 271 (1996) 21808-21813.

[7] G. Mitta, F. Hubert, T. Noël, P. Roch, Myticin, a novel cysteine-rich antimicrobial peptide isolated from haemocytes and plasma of the mussel Mytilus galloprovincialis, Eur. J. Biochem. 265 (1999) 71-78.

[8] A. Pallavicini, M.M. Costa, C. Gestal, R. Dreos, A. Figueras, et al., High sequence variability of myticin transcripts in hemocytes of immune-stimulated mussels suggests ancient host-pathogen interactions, Dev. Comp. Immunol. 32 (2008) 213-226.

[9] M.M. Costa, S. Dios, J. Alonso-Gutiérrez, A. Romero, B. Novoa, et al., Evidence of high individual diversity on myticin C in mussel (Mytilus galloprovincialis), Dev. Comp. Immunol. 33 (2009) 162-170.

[10] M.G. Parisi, H. Li, M. Toubiana, N. Parrinello, M. Cammarata, P. Roch, Polymorphism of mytilin B mRNA is not translated into mature peptide, Mol. Immunol. 46 (2009) 384-392.

[11] J. Zhao, L. Song, C. Li, D. Ni, L. Wu, L. Zhu, et al., Molecular cloning, expression of a big defensin gene from bay scallop Argopecten irradians and the antimicrobial activity of its recombinant protein, Mol. Immunol. 44 (2007) 360-368.

[12] R.D. Rosa, A. Santini, J. Fievet, P. Bulet, D. Destoumieux-Garzón, E. Bachère, Big defensins, a diverse family of antimicrobial peptides that follows different patterns of expression in hemocytes of the oyster Crassostrea gigas, PLoS One 6 (9) (2011) e25594.

[13] M. Gerdol, G. De Moro, C. Manfrin, P. Venier, A. Pallavicini, Big defensins and mytimacins, new AMP families of the Mediterranean mussel Mytilus galloprovincialis, Dev. Comp. Immunol. 36 (2012) 390-399.

[14] M. Gonzalez, Y. Gueguen, G. Desserre, J. de Lorgeril, B. Romestand, E. Bachere, Molecular characterization of two isoforms of defensin from hemocytes of the oyster Crassostrea gigas, Dev. Comp. Immunol. 31 (2007) 332-339.

[15] W. Xu, M. Faisal, Defensin of the zebra mussel (Dreissena polymorpha): molecular structure, in vitro expression, antimicrobial activity, and potential functions, Mol. Immunol. 47 (2010) 2138-2147.

[16] G. Mitta, F. Vandenbulcke, P. Roch, Original involvement of antimicrobial peptides in mussel innate immunity, FEBS Lett. 486 (2000) 185-190.

[17] J.K. Seo, J.M. Crawford, K.L. Stone, E.J. Noga, Purification of a novel arthropod defensin from the American oyster, Crassostrea virginica, Biochem. Biophys. Res. Commun. 338 (2005) 1998-2004.

[18] Y. Gueguen, A. Herpin, A. Aumelas, J. Garnier, J. Fievet, J.M. Escoubas, et al., Characterization of a defensin from the oyster Crassostrea gigas. Recombinant production, folding, solution structure, antimicrobial activities, and gene expression, J. Biol. Chem. 281 (2006) 313-323.

[19] M. De Zoysa, I. Whang, Y. Lee, S. Lee, J.S. Lee, J. Lee, Defensin from disk abalone Haliotis discus discus: molecular cloning, sequence characterization and immune response against bacterial infection, Fish. Shellfish Immunol. 28 (2010) $261-266$.

[20] G.A. Díaz, Defensins and cysteine rich peptides: two types of antimicrobial peptides in marine mollusks, Inv Surv. J. 7 (2010) 157-164.

[21] G. Mitta, F. Vandenbulcke, F. Hubert, P. Roch, Mussel defensins are synthesised and processed in granulocytes then released into the plasma after bacterial challenge, J. Cell Sci. 112 (1999) 4233-4242.

[22] C. Gestal, M. Costa, A. Figueras, B. Novoa, Analysis of differentially expressed genes in response to bacterial stimulation in hemocytes of the carpet-shell clam Ruditapes decussatus: identification of new antimicrobial peptides, Gene 406 (2007) 134-143.

[23] P. Nicolas, D. Vanhoye, M. Amiche, Molecular strategies in biological evolution of antibacterial peptides, Peptides 24 (2003) 1669-1680.

[24] C.A. Semple, P. Gautier, K. Taylor, J.R. Dorin, The changing of the guard: molecular diversity and rapid evolution of beta-defensins, Mol. Divers 10 (2006) 575-584.

[25] A. Padhi, B. Verghese, Molecular diversity and evolution of myticin-C antimicrobial peptide variants in the Mediterranean mussel, Mytilus galloprovincialis, Peptides 29 (2008) 1094-1101.

[26] P. Schmitt, Y. Gueguen, E. Desmarais, E. Bachère, J. de Lorgeril, Molecular diversity of antimicrobial effectors in the oyster Crassostrea gigas, BMC Evol. Biol. 10 (2010) 23.

[27] Q. Ren, M. Li, C.Y. Zhang, K.P. Chen, Six defensins from the triangle-shell pearl mussel Hyriopsis cumingii, Fish. Shellfish Immunol. 31 (2011) 1232-1238.

[28] A. Patil, A.L. Hughes, G. Zhang, Rapid evolution and diversification of mammalian alpha-defensins as revealed by comparative analysis of rodent and primate genes, Physiol. Genomics 20 (2004) 1-11.

[29] N. Pujol, O. Zugasti, D. Wong, C. Couillault, C.L. Kurz, H. Schulenburg, J.J. Ewbank, Anti-fungal innate immunity in C. elegans is enhanced by evolutionary diversification of antimicrobial peptides, PLoS Pathog. 4 (7) (2008) e1000105.

[30] J.A. Tennessen, Molecular evolution of animal antimicrobial peptides: widespread moderate positive selection, J. Evol. Biol. 18 (2005) 1387-1394.

[31] A. Padhi, B. Verghese, S.K. Otta, B. Varghese, K. Ramu, Adaptive evolution after duplication of penaeidin antimicrobial peptides, Fish. Shellfish Immunol. 23 (2007) 553-566.

[32] M. Adhya, H.D. Jeung, H.S. Kang, K.S. Choi, D.S. Lee, M. Cho, Cloning and localization of MCdef, a defensin from Manila clams (Ruditapes philippinarum), Comp. Biochem. Physiol. B 161 (2012) 25-31.

[33] Q. Wang, L. Zhang, J. Zhao, L. You, H. Wu, Two goose-type lysozymes in Mytilus galloprovincialis: possible function diversification and adaptive evolution, PLoS One 7 (9) (2012) e45148.

[34] Q. Wang, C. Wang, C. Mu, H. Wu, L. Zhang, J. Zhao, A novel C-type lysozyme from Mytilus galloprovincialis: insight into innate immunity and molecular evolution of invertebrate C-type lysozymes, PLoS One 8 (6) (2013) e67469.

[35] S. Guindon, J.F. Dufayard, V. Lefort, M. Anisimova, W. Hordijk, O. Gascuel, New algorithms and methods to estimate maximum-likelihood phylogenies: assessing the performance of PhyML 3.0, Syst. Biol. 59 (2012) 307-321.

[36] D. Posada, jModelTest: phylogenetic model averaging, Mol. Biol. Evol. 25 (2008) 1253-1256.

[37] Z. Yang, PAML 4: phylogenetic analysis by maximum likelihood, Mol. Biol. Evol. 24 (2007) 1586-1591.

[38] Z. Yang, W.S.W. Wong, R. Nielsen, Bayes empirical Bayes inferences of amino acid sites under positive selection, Mol. Biol. Evol. 22 (2005) 1107-1118.

[39] X.M. Guo, S.E. Ford, F.S. Zhang, Molluscan aquaculture in China, J. Shell Res. 18 (1999) 19-31.

[40] A. Segarra, J.F. Pépin, I. Arzul, B. Morga, N. Faury, T. Renault, Detection and description of a particular Ostreid herpesvirus 1 genotype associated with massive mortality outbreaks of Pacific oysters, Crassostrea gigas, in France in 2008, Virus Res. 153 (2010) 92-99.

[41] T. Ganz, Defensins and host defense, Science 286 (1999) 420-421.

[42] R. Lai, L.O. Lomas, J. Jonczy, P.C. Turner, H.H. Rees, Two novel non-cationic defensin-like antimicrobial peptides from haemolymph of the female tick, Amblyomma hebraeum, Biochem. J. 379 (2004) 681-685.

[43] M. Sonthi, M. Toubiana, A. Pallavicini, P. Venier, P. Roch, Diversity of coding sequences and gene structures of the antifungal peptide mytimycin (MytM) from the Mediterranean mussel, Mytilus galloprovincialis, Mar. Biotechnol. 13 (2011) 857-867.

[44] K. Peng, J.H. Wang, J.Q. Sheng, L.G. Zeng, Y.J. Hong, Molecular characterization and immune analysis of a defensin from freshwater pearl mussel, Hyriopsis schlegelii, Aquaculture 334 (2012) 45-50.

[45] J. Trowsdale, P. Parham, Mini-review: defense strategies and immunity related genes, Eur. J. Immunol. 34 (2004) 7-17.

[46] T.B. Sackton, B.P. Lazzaro, T.A. Schlenke, J.D. Evans, D. Hultmark, et al., Dynamic evolution of the innate immune system in Drosophila, Nat. Genet. 39 (2007) 1461-1468.

[47] S. Zhu, B. Gao, J. Tytgat, Phylogenetic distribution, functional epitopes and evolution of the CS $\alpha \beta$ superfamily, Cell Mol. Life Sci. 62 (2005) 2257-2269.

[48] M. Vera, P. Martínez, L. Poisa-Beiro, A. Figueras, B. Novoa, Genomic organization, molecular diversification, and evolution of antimicrobial peptide myticin-C genes in the mussel (Mytilus galloprovincialis), PLoS One 6 (8) (2011) e24041.

[49] T. Schneider, T. Kruse, R. Wimmer, I. Wiedemann, V. Sass, U. Pag, et al, Plectasin, a fungal defensin, targets the bacterial cell wall precursor lipid II, Science 328 (2010) 1168-1172.

[50] P. Schmitt, M. Wilmes, M. Pugnière, A. Aumelas, E. Bachère, H.G. Sahl, et al Insight into invertebrate defensin mechanism of action: oyster defensins inhibit peptidoglycan biosynthesis by binding to lipid II, J. Biol. Chem. 38 (2010) 29208-29216. 